\title{
On the Calculation and Measurement of Mean Room Surface Exitance
}

James Duff

Technological University Dublin, james.duff@arup.com

G. Antonutto

Arup Lighting, London, United Kingdom

S. Torres

Arup Lighting, London, United Kingdom

Follow this and additional works at: https://arrow.tudublin.ie/engscheleart2

Part of the Electrical and Electronics Commons

\section{Recommended Citation}

Duff, J., Antonutto, G. \& Torres, S. (2015) On the Calculation and Measurement of Mean Room Surface Exitance, Lighting Research and Technology 48(3) · July 2015 DOI: 10.1177/1477153515593579

This Article is brought to you for free and open access by the School of Electrical and Electronic Engineering at ARROW@TU Dublin. It has been accepted for inclusion in Articles by an authorized administrator of ARROW@TU Dublin. For more information, please contact arrow.admin@tudublin.ie, aisling.coyne@tudublin.ie, gerard.connolly@tudublin.ie.

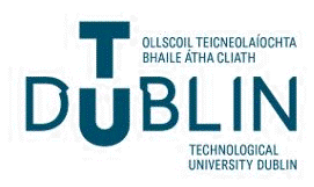




\title{
On the calculation and measurement of mean room surface exitance
}

\author{
J Duff BSc ${ }^{\mathrm{a}}$, G Antonutto $\mathrm{MSc}^{\mathrm{b}}$ and S Torres $\mathrm{PhD}^{\mathrm{b}}$ \\ a Arup Lighting and Dublin Institute of Technology, Dublin, Ireland \\ b Arup Lighting, London, United Kingdom \\ Short title: Calculation and measurement of MRSE
}

Received 1 April 2015; Revised 11 May 2015; Accepted

Cuttle has proposed mean room surface exitance as a metric for use within interior lighting design. Two reported barriers to its use are; a lack of available software to calculate the metric and the tedious and cumbersome nature of recording its values in the field using current equipment and methodologies. This paper presents a method for facilitating the calculation of mean room surface exitance using readily available freeware, namely Radiance, and also presents a methodology that utilises high dynamic range imaging as a pragmatic alternative for field measurement.

Address for correspondence: James Duff, 50 Ringsend Road, Dublin 4, Ireland.

\section{Introduction}

Over the past decade, Cuttle has been the prime advocate for reforming internal lighting standards from their current state, to consider a more holistic design approach that better relates to what we see. ${ }^{1-7}$ Central to Cuttle's proposal is the use of mean room surface 
exitance (MRSE), this being a measure of overall density of reflected luminous flux within a space. Through a thought experiment, ${ }^{3}$ Cuttle defines MRSE as:

$$
M R S E=\frac{F R F}{A_{\propto}}
$$

Where $F R F$ is the first reflected luminous flux, being the sum of the direct luminous flux reflected from each surface of area $A_{s}$ and reflectance $\rho_{s}$ :

$$
F R F=\sum E_{S(d)} A_{S} \rho_{S}
$$

And $A \alpha$ is the room absorptance, being the sum of the surface areas times their absorptance values, where absorptance is given by one minus reflectance:

$$
A \alpha=\sum A_{S}\left(1-\rho_{S}\right)
$$

Whilst equation (1) offers a theoretically simple way to calculate MRSE, derivation of the first reflected luminous flux component can be a little more tricky in reality. In addition, the formula only holds true for spaces with identical surface reflectance properties. ${ }^{8}$ If an assumption is made that all surfaces within a space are lambertian diffusers, then both these items can be overcome, with MRSE being calculated as follows.

For each surface within a space, the mean exitance of that surface is given by the product of the mean luminance and pi:

$$
M_{S}=L_{S} \pi
$$

The MRSE is given by the sum of the area weighted exitance values for all surfaces, divided by the total room surface area.

$$
M R S E=\frac{\sum M_{S} A_{S}}{\sum A_{S}}
$$


Cuttle’s ideas have generally been well received, ${ }^{9-14}$ but two commonly heard criticisms, and echoed by Boyce, ${ }^{15}$ are that it cannot be calculated with current software and cannot be measured in the field. This paper introduces a method that utilises Radiance ${ }^{16}$ software as a platform to calculate MRSE and high dynamic range (HDR) imaging to estimate levels of MRSE in the field.

\section{Calculation of mean room surface exitance}

\subsection{Procedure}

Radiance is a suite of programs for the analysis and visualisation of lighting design. It lacks a graphical user interface, but as such, offers the user greater flexibility than typical lighting simulation programs. The authors have developed a script to calculate MRSE for electric lighting designs. This is available to download from a web link given at the end of this paper. To run the script, users will require an OS $\mathrm{X}$ interface with a full suite of Radiance commands installed, along with the ability to run a range of commands in the Perl language.

In general, the script works in two parts. The first applies calculation grids to each surface in the space and calculates a mean exitance value for it, as given in equation (4). The second processes the results to produce the MRSE, as given in equation (5). The individual steps to run the script are:

1. Create the 3D geometry as normal with any electric lighting Radiance calculation.

2. Create a rad_X folder which houses surfaces that are to have exitance calculations applied, i.e. excluding all light, glass, etc. types. $X$ is a model reference number which is called within the command line. 
3. To run the script, type ./run_mrse.pl $X Y$ with the command line, where $X$ is the integer model reference number and $Y$ is an optional float that sets the distance apart for which calculation points will be applied. The default float is $200 \mathrm{~mm}$.

4. To produce the MRSE, within the command line type total $-m$ tmp_X/all.res. This will spit out the MRSE for the space.

\subsection{Validation}

To examine the accuracy of the script, a trial was conducted where results computed with it were compared with real-world measurements and also triangulated with calculations conducted using a radiosity based software. The space was $5000 \mathrm{~mm}$ long, $2900 \mathrm{~mm}$ wide and $2850 \mathrm{~mm}$ high, contained two ceiling mounted linear fluorescent luminaires and no furniture. The luminaires were dimmed to a level such that the real-world measurements produced an MRSE of approx. $100 \mathrm{~lm} / \mathrm{m}^{2}$. The value of the luminous flux emitted from each luminaire in the dimmed state was then calculated using the manufacturer's dimming curve and cross-referenced with in-situ illuminance measurements. The total output luminous flux used in the software calculations was then based on this figure. The validation process was as follows:

- Calculation using the script was as described previously, using the default float and five ambient bounces (- $a b 5)$.

- Calculation within the radiosity software was completed using the default indirect calculation settings to derive luminance values on each room surface, then post processing these to obtain the MRSE using equations (4) and (5).

- The real-world calculation was carried out using a luminance meter and equations (4) and (5). A grid point spacing of 300mm was used.

The results of each calculation are given in Table 1. 


\section{Measurement of mean room surface exitance}

HDR imaging is a set of techniques used in photography to produce a wider dynamic range of luminosity than is typically possible using standard digital imaging or photography techniques. Essentially, HDR imaging uses multiple exposures of the same scene to produce images that better represent the perceived luminous environment. At present, this can be

applied to produce luminance-calibrated images of the lit environment. ${ }^{17,18}$ This procedure has been adapted by the authors to calculate the indirect flux incident on the camera lens. The intention being that multiple views of a space from various angles are captured, with the mean of the values of indirect luminous flux being equivalent to the MRSE.

\subsection{Procedure}

HDR images can be recorded using a digital single-lens reflex camera and calibrated using available software such as Photosphere or using hdrgen and Radiance. ${ }^{16,19}$ The authors have developed a script to estimate the indirect luminous flux incident on a camera lens and this is available to download from a web link given at the end of this paper. In general, it works with the user manually defining the value of direct luminance incident on the camera lens and removing all pixels in excess of this value from the HDR image, with a calculation of indirect illuminance at the camera lens conducted once this is complete. The necessary steps to apply this method are as follows:

1. Capture a HDR image using an appropriate camera.

2. Calibrate the image using either Photosphere or hdrgen and Radiance.

3. Open the image using $X 11$ or a similar program that allows individual pixel luminance values to be viewed. While doing so, note the lowest quantity of direct luminance; this is used as the threshold above which pixels containing higher luminances are excluded. 
4. In the command line, type ./run -t $X-p Y$, where $X$ is the threshold luminance value above which pixels will be excluded and $Y$ is the filename of the HDR image to which the script will be applied. The value of indirect luminous flux is then spat out in the command line and should be noted.

5. As a cross-check, .tif images indicating both the direct and diffuse calculated components are generated. These can be viewed to ensure that the appropriate pixels have been excluded from the calculation.

6. The above process is repeated for a number of views within a space and the mean value of each indirect illuminance is representative of the MRSE. The mean must be manually calculated.

\subsection{Validation}

To examine the accuracy of this method, a trial was conducted where results computed with it were compared with field measurements and also triangulated with calculations carried out in Radiance. The room and set-up described previously were used. The validation process was as follows:

- With the lens available to the authors at the time, to fully capture the entire space and make the calculation appropriately accurate a total of eight HDR images were recorded. Two from each corner of the space directly facing the opposing corner but at plus and minus $45^{\circ}$ to the horizontal respectively. Using $X 11$, direct luminance values were derived for each image based on the range of luminances presented in the camera view. The mean of the indirect flux within all eight images was taken as the MRSE. It should be noted that if a lens with an appropriate view angle (such as a wide angle or fish-eye) had been available, the number of HDR images could have been reduced to two, with similar levels of accuracy maintained. 
- Calculation in Radiance was carried out using the script and procedure described previously in this paper.

- The in-field calculation was carried out using a luminance meter and equations (4) and (5). A gird point spacing of 300mm was used.

The results of each calculation are given in Table 2.

\section{Discussion and limitations}

Recommended settings for a typical Radiance calculation are suggested within a number of publications, ${ }^{20,21}$ but when applying the authors' calculation script, the quantity of ambient bounces $(-a b)$ applied is of particular importance. Within Radiance, the number of ambient bounces applied governs the number of inter-reflections calculated. As a metric, MRSE theoretically requires an infinite number of flux inter-reflections, which is not practical given the computing power available to a typical lighting consultant. The authors recommend four to five ambient bounces to be a good trade-off between accuracy and calculation time, but this may need to change when extreme levels of surface reflectance are encountered.

When applying the measurement method described, the user must define a direct luminance threshold value. This could be considered a limitation as the absolute precise value above which direct luminance is incident on the camera lens is not so easy to determine and can be difficult under certain circumstances. For example, consider an oddly shaped prismatic diffuser with very high output lamps hidden behind; here it is difficult to distinguish between the direct and indirect luminance incident on the camera lens as a result of this luminaire. Consequently, the variability in choosing the value of threshold luminance has an impact on the returned value of indirect illuminance. In addition, on occasion, lighting designers will encounter situations where the value of indirect luminance within a space is 
actually greater than the value of direct luminance in the same field of view. As an extreme example, consider a high output wall-washing luminaire placed far too close to a wall and in the same field of view, a decorative, dimmed, low-output incandescent lamp. The method developed by the authors cannot exclude the direct flux from the dim incandescent lamp whilst including the high indirect luminance levels as a results of the wallwash. Whilst this is an extreme example, the experience of the authors is that less severe versions of this situation are encountered more regularly than might be expected; a typical example being a direct/indirect type luminaire suspended close to a ceiling. Here, the value of indirect luminance on a white ceiling above can often be very similar, if not in excess, of the direct luminance at a given angle to the horizontal below.

\section{Conclusions}

The authors have introduced a procedure to calculate MRSE within software and measure its value in the field. Neither of these procedures are intended to be globally adopted, but rather they serve as proof of concept that both are currently possible and could be easily implemented by software developers for mass use in the future.

\section{Downloads}

To download the scripts produced by the authors, please use the link below.

\section{https://www.dropbox.com/l/lC62txkbVpcW1AQ8HPfydu}




\section{References}

1. Cuttle C. Lighting by Design, 2st edition, Oxford, Architectural Press, 2008.

2. Cuttle C. Lighting Design: A Perception-Based Approach, London, Routledge, 2015.

3. Cuttle C. Towards the third stage of the lighting profession. Lighting Research and Technology, March 2010; vol. 42, 1: pp. 73-93.

4. Cuttle C. Perceived adequacy of illumination: A new basis for lighting practice. Proceedings of the 3rd Professional Lighting Design Convention, PLDA, Madrid, 2011.

5. Cuttle C. A new direction for general lighting practice. Lighting Research and Technology, February 2013; vol. 45, 1: pp. 22-39.

6. Cuttle C. Correspondence: The lumen dumper's solution. Lighting Research and Technology, June 2013; vol. 45, 3: pp. 391-393.

7. Cuttle C. Redefining the Purpose of General Lighting Practice. Proceedings of the LS-14 Symposium, June 22-27 2014; pp. 121-130.

8. Raynham, P. Room lighting in the absence of a defined visual task and the impact of mean room surface exitance. Lighting Research and Technology, first published on November 27, 2014, doi:10.1177/1477153514561071.

9. Venning B, Poulton K, Shaw K, Loe DL, Raynham P, Hoggett N. Cuttle’s Theory, the profession responds. Society of Light and Lighting Newsletter. Vol3, Iss 1. Jan/Feb 2010. pp 7-9.

10. Macrae I. Comment 1: A new direction for general lighting practice. Lighting Research and Technology, February 2013; vol. 45, 1: pp. 22-39.

11. Brandston HM. Comment 3: Towards the third stage of the lighting profession. Lighting Research and Technology, March 2010; vol. 42, 1: pp. 73-93.

12. Wilde, MB. Comment 2: A new direction for general lighting practice. Lighting Research and Technology, February 2013; vol. 45, 1: pp. 22-39. 
13. Bedocs L. Comment 1: Towards the third stage of the lighting profession. Lighting Research and Technology, March 2010; vol. 42, 1: pp. 73-93.

14. Mansfield, KP. Comment 2: Towards the third stage of the lighting profession. Lighting Research and Technology, March 2010; vol. 42, 1: pp. 73-93.

15. Boyce, PR, Lighting quality for all? Proceedings of the SLL International Lighting Conference, Dublin, April 2013.

16. Ward G, Shakesphere R. Rendering with Radiance. San Francisco: M. Kaufmann, 2004.

17. Inanici MN. Evaluation of high dynamic range photography as a luminance data acquisition system. Lighting Research and Technology, 2006; 38(2): 123-134.

18. Mardaljevic J, Painter B, Andersen M. Transmission illuminance proxy HDR imaging: A new technique to quantify luminous flux. Lighting Research and Technology, 2009; 41(1): 27-49.

19. Ward G. Photosphere. Retrieved 04 June 2015 from /http://www.anyhere.com/

20. Ward G. The RADIANCE lighting simulation and rendering system. Proceedings of SIGGRAPH 94: Computer Graphics Annual Conference Series. Orlando, FL: Association for Computing Machinery's Special Interest Group on Computer Graphics, 1994: pp.459-472.

21. Ward G, Rubinstein F, Clear R. A ray tracing solution for diffuse inter-reflection. Computer Graphics 1988; 22: 85-92. 
Table Error! Main Document Only.. Calculation results showing levels of mean room surface exitance derived using the three methods described previously.

\begin{tabular}{|c|c|}
\hline Calculation Method & Mean Room Surface Exitance $\left(\mathrm{lm} / \mathrm{m}^{2}\right)$ \\
\hline Radiance script & 101 \\
\hline Radiosity software & 107 \\
\hline Luminance meter measurements & 103 \\
\hline
\end{tabular}

Table Error! Main Document Only.. Calculation results showing levels of mean room surface exitance derived using the three methods described previously.

\begin{tabular}{|l|c|}
\hline Calculation Method & Mean Room Surface Exitance (lm/m²) \\
\hline HDR images & 111 \\
\hline Radiance script & 101 \\
\hline Luminance meter measurements & 103 \\
\hline
\end{tabular}

